\title{
Performance Comparison of Particle Swarm Optimization and Gravitational Search Algorithm to the Designed of Controller for Nonlinear System
}

\author{
Sahazati Md Rozali, ${ }^{1}$ Mohd Fua'ad Rahmat, ${ }^{2}$ and Abdul Rashid Husain ${ }^{2}$ \\ ${ }^{1}$ Department of Control System and Automation, Faculty of Electrical Engineering, Universiti Teknikal Malaysia Melaka, \\ 76100 Durian Tunggal, Melaka, Malaysia \\ ${ }^{2}$ Department of Control and Mechatronics Engineering, Faculty of Electrical Engineering, Universiti Teknologi Malaysia, \\ 81310 Skudai, Johor, Malaysia
}

Correspondence should be addressed to Mohd Fua'ad Rahmat; fuaad@fke.utm.my

Received 14 November 2013; Revised 18 February 2014; Accepted 25 February 2014; Published 14 April 2014

Academic Editor: Hak-Keung Lam

Copyright (C) 2014 Sahazati Md Rozali et al. This is an open access article distributed under the Creative Commons Attribution License, which permits unrestricted use, distribution, and reproduction in any medium, provided the original work is properly cited.

\begin{abstract}
This paper presents backstepping controller design for tracking purpose of nonlinear system. Since the performance of the designed controller depends on the value of control parameters, gravitational search algorithm (GSA) and particle swarm optimization (PSO) techniques are used to optimise these parameters in order to achieve a predefined system performance. The performance is evaluated based on the tracking error between reference input given to the system and the system output. Then, the efficacy of the backstepping controller is verified in simulation environment under various system setup including both the system subjected to external disturbance and without disturbance. The simulation results show that backstepping with particle swarm optimization technique performs better than the similar controller with gravitational search algorithm technique in terms of output response and tracking error.
\end{abstract}

\section{Introduction}

As similar as other control methods, backstepping can be used for tracking and regulating the problem. The backstepping method permits to obtain global stability in the cases when the feedback linearization method only secures local stability [1]. The fundamental concept of backstepping method is introduced in $[2,3]$. The backstepping method was used in numerous applications such as flight trajectory control [4], industrial automation systems, electric machines, nonlinear systems of wind turbine-based power production, and robotic system. It is also shown to be an effective tool in adaptive control design for estimating parameters [5] and optimal control problems. In addition, the observer based on backstepping technique is also designed for force control of electrohydraulic actuator system [6]. This control strategy guarantees the convergence of the tracking error. This control technique is also used as an observer that combined with adaptive and sliding mode controller to control DC servo motor [7] and controller for electrohydraulic active suspension system [8].

A robust state-feedback controller is designed by employing backstepping design technique such that the system output tracks a given signal and all signals in the closedloop system remain bounded [9]. The backstepping design strategy also used to develop a Lyapunov-based nonlinear controller for a hydraulic servo system which incorporates load, hydraulic, and valve dynamics in the design process [10]. In addition, combination of backstepping with variable structure and adaptive controller for plants with relative degree one is presented using input/output measurements [11]. Switching laws are used to increase robustness to parametric uncertainties and disturbances and improve transient response of the system. Adaptive backstepping is also designed for a kind of servo system in a flight simulator [12]. The controller is developed to overcome the parameter uncertainties and load disturbances in the system. Backstepping is also integrated with adaptive controller in [13]. In this paper, 
friction is modelled as Lu Gre friction and it is combined with external force. Both of these friction and external force are considered as uncertainties and adaptive scheme is suited to overcome this uncertainties. Backstepping is also used as an adaptive method for strict-feedback nonlinear systems by using multilayered neural networks [14]. The developed control scheme introduced modified Lyapunov function for first-order plant by having a smooth and free-singularity adaptive controller Backstepping controller is designed for position and force tracking of electrohydraulic servos system [15]. In this work, the model of the system is considered as third, fourth and five states and backstepping controller is designed for each of these states. Besides, backstepping is also used in position tracking of an electropneumatic system [16]. It is known that backstepping is suitable for strict feedback system only. However this paper proved that this type of controller can be applied to the type of the system which is not a strict-feedback system. Generally, small scale of helicopter is highly nonlinear, coupled, and sensitive which caused difficulty in controlling task. However, a nonlinear adaptive backstepping control is proposed for this system by focusing vertical flight motion of the system [17]. Backstepping controller is also integrated with sliding mode control technique and is proposed for controlling underactuated systems [18]. In this research, backstepping algorithm helps the system to immune with matched and mismatched uncertainties while sliding mode control provides robustness.

The control parameter of backstepping is very important in order to achieve performance target. Thus, it can be obtained by several methods such as heuristic approach, artificial intelligent technique, and optimization algorithm. Based on heuristic method where at some points the incorporation of desired system performance at design stage is difficult to be integrated. Ye used neural network to find the parameter of backstepping controller in order to improve the tracking performance of mobile robots [19], while fuzzy logic and least mean square are used for parameter tuning for backstepping controller to stabilize the attitude of quadrotor UAV [20]. Other papers tuned the controller parameters by optimisation techniques. These can be seen when ant colony optimization algorithm is used in [21]. This research used combination of fuzzy logic controller and neural network to acquire parameters of backstepping and ant colony optimization technique is used to attain the best value for parameters of fuzzy neural network. As an approach to ship course control, backstepping is developed with genetic algorithm technique to optimise its parameter [1]. The similar algorithm is also used in designing backstepping for flight control system [22]. Based on reviews, particle swarm optimization (PSO) is the most technique that is applied to be combined with backstepping controller in order to adjust its control parameters [23-27]. PSO is used to tune backstepping controller for power system stability enhancement [23]. The proposed technique shows that the designed controllers are effective in stabilizing the system under severe contingencies and perform better than conventional power system stabilizers.

PSO is also used for backstepping parameter tuning for maglev transportation system [24] and the online levitated balancing and propulsive positioning of a magnetic-levitation transportation system [25]. The proposed algorithm is proved to be more effective than the standard backstepping control itself. In addition, PSO is also integrated with backstepping technique to design speed controller for permanent magnet synchronous motor based on adaptive law [26]. The parameter of controller is tuned by using PSO. Simulation results show that the controller has robust and good dynamic response. An adaptive backstepping control technique with acceleration feedback is designed in order to reject the uncertainties and external disturbances for Dynamic Positioning System with slowly varying disturbances [27]. In this study, the controller parameters and acceleration feedback parameters are optimized using PSO.

Gravitational search algorithm (GSA) is among the latest optimisation technique and there is no research that combines this technique with backstepping controller. Thus, it is chosen to be assimilated with this controller such that the control parameter is adjusted automatically based on the system requirements. Performance of gravitational search algorithm (GSA) is compared with particle swarm optimization (PSO) technique for optimal tuning of PI controllers dedicated to a class of second-order processes with integral component and variable parameters [28]. The results show that both solutions demonstrate good convergence. GSA is also used to determine the parameters of PID controller for speed and position control of DC motor [29]. Mean squared error (MSE) performance index has been used as an objective function in this work. The effectiveness of proposed method is compared with Ziegler-Nichols method in speed control DC motor. Adaptive gravitational search algorithm is presented for the optimal tuning of fuzzy controlled servo system characterized by second-order models with an integral component and variable parameters [30]. The proposed method results a new generation of Takagi-Sugeno proportional-integral fuzzy controllers with a reduced time constant sensitivity. Besides, the parameters of sensor monitoring selection for each round in a point coverage network had been optimised using GSA [31]. Simulation results show that the method is superior to former algorithms in the aspects of parameters optimization, lifetime increase, and energy consumptions. Fuzzy GSA miner is introduced to develop a novel data mining technique [32]. In the research, fuzzy controller is designed as adaptive control for the gravitational coefficient then Fuzzy-GSA is employed to construct a novel data mining algorithm for classification rule discovery from reference data set.

This research work focused on designing backstepping controller for position tracking of nonlinear system. Electrohydraulic actuator system (EHA) is chosen as numerical example since its position tracking is highly nonlinear [15]. This paper is different with [13] in terms of additional signal as perturbation to the actuator of the system. The control parameters of backstepping controller are then tuned by using gravitational search algorithm (GSA) and particle swarm optimization (PSO) techniques in order to acquire the suitable values of its control parameters for accurate tracking response. GSA is chosen since this technique has never been applied to be integrated with backstepping controller in order 
to tune its control parameters. Combination of backstepping with PSO has not been applied yet to electrohydraulic actuator system. Besides, PSO has remarkably few parameters to adjust and it has been used for approaches that can be used across a wide range of applications [33]. However, since most of reviews on backstepping assimilate the controller with PSO, the performance of combination of backstepping with GSA is compared with the integration of backstepping with PSO for this system. This is another extra contribution in this research compared to [15]. The performance of the designed controller with these optimization techniques is compared in terms of tracking error. Sum of squared error (SSE) is used as an objective function for both techniques. The effectiveness of the backstepping controller is verified in simulation environment under various system setup including both the system subjected to external disturbance and without disturbance.

\section{Problem Formulation}

Consider state-space model of EHA system is given as follows [13]:

$$
\begin{gathered}
\dot{x_{1}}=x_{2}, \\
\dot{x_{2}}=-\frac{k}{m} x_{1}-\frac{f}{m} x_{2}+\frac{S}{m} x_{3}-\frac{F_{L}}{m}, \\
\dot{x_{3}}=-\frac{S}{k_{c}} x_{2}-\frac{k_{l}}{k_{c}} x_{3}+\frac{c}{k_{c}} \sqrt{\frac{p_{a}-x_{3}}{2}} k_{v} u,
\end{gathered}
$$

with

$$
c=c_{d} w \sqrt{\frac{2}{\rho}} .
$$

$x_{1}=$ displacement of the load $(\mathrm{cm}), x_{2}=$ load velocity $(\mathrm{cm} / \mathrm{s}), x_{3}=$ pressure difference $p_{1}-p_{2}$ between the cylinder chambers caused by load $(N), F_{L}=$ external disturbance given to the system, and $* F_{L}$ can be constant or time-varying disturbance.

Backstepping controller designed is started with defining error for each state $x_{1}, x_{2}$, and $x_{3}$, respectively, as

$$
e_{i}=x_{i}-x_{i d}
$$

with $i=1,2,3$ is the error for each state, $x_{1 d}=$ reference input and $x_{2 d}$ and $x_{3 d}=$ virtual control.

The control objective is to have EHA track of a specified $x_{1 d}$ position trajectory so that $e_{1} \rightarrow 0$.

Proposition 1. Equation (1) is assumed with nonsaturating load which means that $x_{3}<P_{a}$. Let $k_{1}, k_{2}, k_{3}, \rho_{1}, \rho_{2}$, and $\rho_{3}$ be positive tuning parameters, the best and asymptotically stabilized position tracking of (1) with respect to the desired
TABLE 1: Parameter of EHA system.

\begin{tabular}{lc}
\hline Load at the EHS rod, $m$ & $0.33 \mathrm{Ns}^{2} / \mathrm{cm}$ \\
Piston area, $S$ & $10 \mathrm{~cm}^{2}$ \\
Coefficient of viscous friction, $f$ & $27.5 \mathrm{Ns} / \mathrm{cm}$ \\
Coefficient of aerodynamic elastic force, $k$ & $1000 \mathrm{~N} / \mathrm{cm}$ \\
Valve port width, $w$ & $0.05 \mathrm{~cm}$ \\
Supply pressure, $P_{a}$ & $2100 \mathrm{~N} / \mathrm{cm}^{2}$ \\
Coefficient of volumetric flow of the valve & 0.63 \\
port, $c_{d}$ & \\
Coefficient of internal leakage between the & $2.38 \times 10^{-3} \mathrm{~cm}^{5} / \mathrm{Ns}$ \\
cylinder chambers, $k_{l}$ & $0.017 \mathrm{~cm}_{l} / \mathrm{V}$ \\
Coefficient of servo valve, $k_{v}$ & $2.5 \times 10^{-4} \mathrm{~cm}^{5} / \mathrm{N}$ \\
Coefficient involving bulk modulus and & \\
EHA volume, $k_{c}$ & $8.87 \times 10^{-7} \mathrm{Ns}^{2} / \mathrm{cm}^{4}$ \\
Oil density, $\rho$ &
\end{tabular}

input can be achieved by the control $u$ and virtual control $x_{2 d}$ and $x_{3 d}$ given by

$$
\begin{gathered}
u=\frac{k_{c}}{\rho_{3} c k_{v}} \sqrt{\frac{2}{P_{a}-x_{3}}} \\
\times\left[-\frac{\rho_{2}}{m} S e_{2}+\frac{\rho_{3}}{k_{c}} S x_{2}+\frac{\rho_{3}}{k_{c}} k_{l} x_{3}+\rho_{3} x_{3 d}^{\cdot}-k_{3} e_{3}\right], \\
x_{3 d}=\frac{1}{S}\left[k x_{1}+f x_{2}-\frac{\rho_{1}}{\rho_{2}} m e_{1}+m x_{2 d}^{\cdot}-\frac{k_{2}}{\rho_{2}} m e_{2}+F_{o}\right], \\
x_{2 d}=\dot{x_{1 d}}-k_{1} e_{1} .
\end{gathered}
$$

Equation (4) is obtained by taking derivative of Lyapunov functions for each state of EHA system so that by substituting these equation to the derivative of Lyapunov functions, the functions will be negative definite in order to assure the stability of the designed controller. The Lyapunov functions for each state $x_{1}, x_{2}$, and $x_{3}$ are given as follows:

$$
\begin{gathered}
V_{1}=\frac{\rho_{1}}{2} e_{1}^{2}, \\
V_{2}=V_{1}+\frac{\rho_{2}}{2} e_{2}^{2}, \\
V_{3}=V_{2}+\frac{\rho_{3}}{2} e_{3}^{2} .
\end{gathered}
$$

In order to obtain good tracking performance, the control parameters of backstepping controller, $k_{1}, k_{2}, k_{3}, \rho_{1}, \rho_{2}$, and $\rho_{3}$ should be chosen carefully. In this work, particle swarm optimization (PSO) and gravitational search algorithm (GSA) are used to adjust these parameters so that the suitable value can be acquired to reduce the tracking error between reference input and system output.

Case 1 (constant disturbance). Constant value of signal $F_{L}=$ $10000 \mathrm{~N}$ is added as perturbation to system actuator. Let the system parameter as shown in Table 1, by substituting the control signal in (4) to the derivative of (5), the output of the 
chosen system is proved to be asymptotically stable by having derivative of Lyapunov functions as follows:

$$
\begin{gathered}
\dot{V}_{1}=-k_{1} \rho_{1} e_{1}^{2}+\rho_{1} e_{1} e_{2}, \\
\dot{V}_{2}=-k_{1} \rho_{1} e_{1}^{2}-k_{2} e_{2}^{2}+\frac{\rho_{2}}{m} S e_{2} e_{3} \\
\dot{V}_{3}=-k_{1} \rho_{1} e_{1}^{2}-k_{2} e_{2}^{2}-k_{3} e_{3}^{2} .
\end{gathered}
$$

Case 2 (step signal as disturbance). Step input signal is given as an external disturbance, $F_{L}$ to the system actuator. Repeating similar process as in Case 1, the objective is to design controller for the system such that the closed-loop system is stable.

Case 3 (time-varying signal as disturbance). In order to assure the robustness of the designed controller, external disturbance $F_{L}$ is replaced with time-varying signal given by

$$
F_{L}=0.2 e^{-t}+0.2 e^{-t} \cos \left(2.1794 t-167^{\circ}\right) \text {. }
$$

Equivalent steps in the previous cases are replicated in this case to reassure the stability of closed-loop system with the given perturbation.

Case 4 (no disturbance). In this case, $F_{L}=0$. This case is taken as benchmark for other previous cases.

\section{Algorithm}

Particle swarm optimization (PSO) algorithm is a population-based search algorithm based on the simulation of the social behaviour of birds within a flock [34]. This algorithm optimizes a problem by having a population of candidate solutions and moving these particles around in the search-space according to simple mathematical formulae over the particle's position and velocity. The particle's movement is influenced by its local best known position and is also guided toward the best known positions in the search-space which are better positions found by other particles. Therefore, the swarm is expected to move toward the best solutions.

Gravitational search algorithm (GSA) is developed by Rashedi et al. [35] based on the law of gravity and mass interactions. In this algorithm, the searcher agents are a collection of masses which interact with each other based on the Newtonian gravity and the laws of motion. Objects are considered as agents. The performance of agents is measured by their masses. They are attracted to each other by the gravity force which causes a global movement of all objects toward the objects with heavier masses. In GSA, each agent has four specifications; position, inertial mass, active gravitational mass, and passive gravitational mass. The position of the mass corresponds to a solution of the problem. Figure 1 illustrates the block diagram of the proposed backstepping controller with PSO or GSA algorithm.

This paper is different from [15] in terms of different types of external disturbance given to the system. For all the cases mentioned before, backstepping controller is designed for

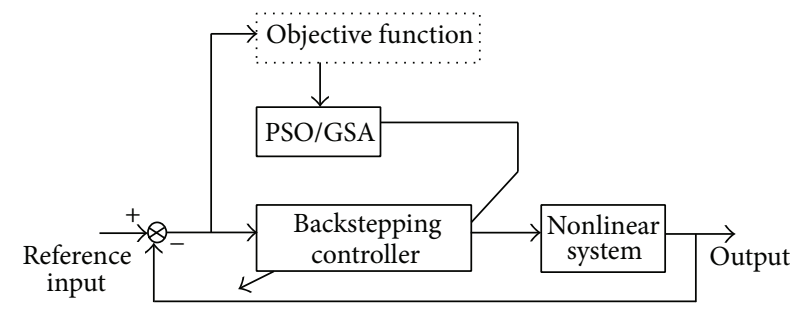

FIGURE 1: Block diagram of the proposed backstepping controller with PSO/GSA algorithm.

each case. Instead of using trial and error method as shown in [15] to set the value of control parameters, $k_{1}, k_{2}, k_{3}, \rho_{1}$, $\rho_{2}$, and $\rho_{3}$, this research work applied optimization process as a tool to acquire the suitable value for these parameters so that good tracking performance can be achieved. GSA is chosen to optimize these parameters since combination of backstepping with GSA has not been tried yet. Most reviews on backstepping controller tuned its parameter by using PSO [23-27]. Therefore, the performance of combination of backstepping with GSA is then compared to integration of similar controller with PSO. The objective is to get good tracking performance with small tracking error.

\section{Simulation Results}

The basic GSA parameters are $G_{-} T=10$, Bheta $=0.7$, and Epsilon $=G_{-} T$ with number of agents, $N=10$ within iteration, $T=20$. With similar number of particles, $N_{p}=10$ within same iteration, $N_{i}=20$, basic parameters of PSO is chosen as $s=c=1.42$ and inertia weight, $w=0.9$. These values are standard values that are always used for these two optimization techniques. The performance of the designed controller with both techniques is compared in terms of tracking error. Sum of squared error (SSE) is used as an objective function. The formula of SSE is given by

$$
\mathrm{SSE}=\sum_{i=1}^{n}\left(x_{i}-y_{\mathrm{ref}}\right)^{2} \text {, }
$$

where SSE $=$ sum of squared error, $i=$ number of iteration, $x_{i}=$ system output at $i$ iteration, and $y_{\text {ref }}=$ reference input.

A good tracking response will give small value of SSE. The effectiveness of the combination of backstepping controller with these two optimization techniques is verified in simulation environment under various system setup including both the system subjected to external disturbance and without disturbance. Nonlinear system chosen in this work which is electrohydraulic actuator (EHA) system is a tracking system. Thus, the aim of control of this system is to have a good tracking of the specified desired position of reference input with small tracking error. The parameter of the testing system is shown in Table 1 [15].

Case 1. Figure 2 illustrates the system output with respect to reference input given to the system and position tracking error between reference input and system output for each 


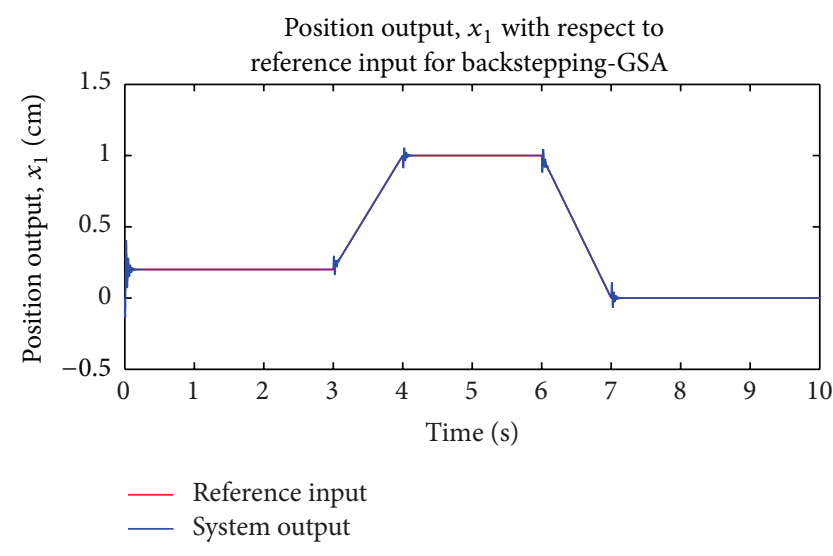

(a)

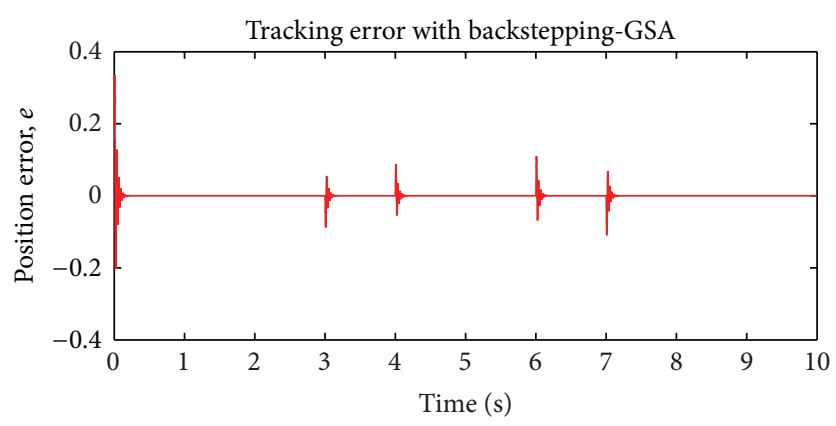

(c)

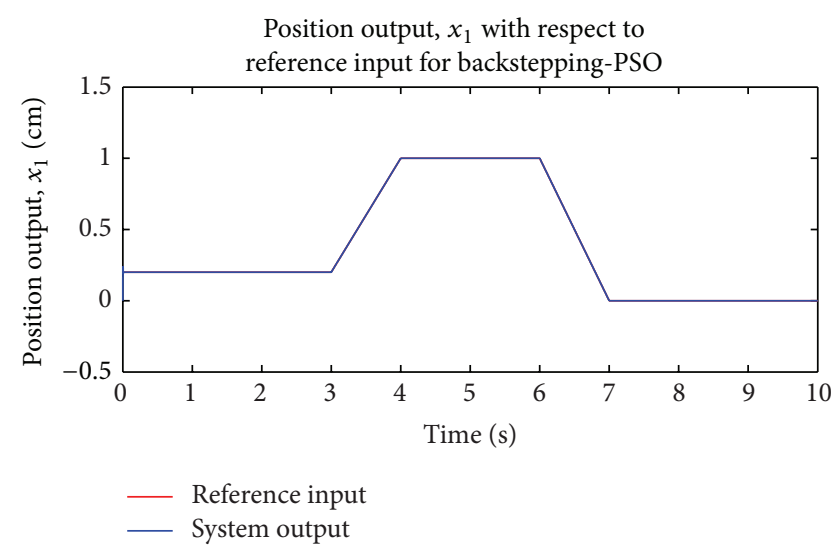

(b)

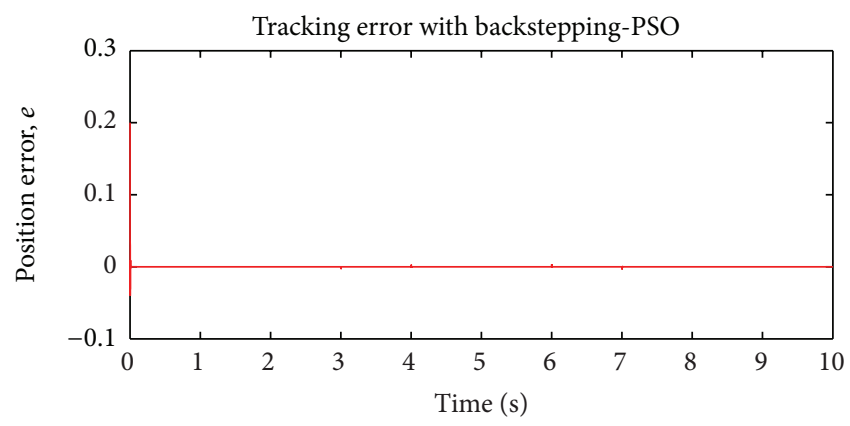

(d)

FIGURE 2: Position output with respect to reference input given and tracking error for backstepping-PSO and backstepping-GSA with constant disturbance injected to the system.

combination of backstepping with GSA and PSO, respectively.

Based on Figure 2, both the system output at the top of the figure yielded by backstepping-PSO and backsteppingGSA track the reference input given with small error. However, backstepping-GSA produces oscillate output while backstepping-PSO generates smooth tracking response. This oscillation can be seen obviously at each corner of the output yielded by backstepping-GSA. The oscillation also can be seen in the error signal created by backstepping-GSA although the value of tracking error is small and almost zero. This is shown by the bottom graphs in the figure.

Case 2. System output with respect to reference input given and tracking error between system output and reference input for both integration of backstepping with PSO and GSA for this case are illustrated in Figure 3.

By referring to Figure 3, when step signal is given as perturbation to the system, the top graphs of the figure show that both backstepping-GSA and backstepping-PSO produce smooth output tracking with respect to reference input given. However, backstepping-GSA generates bigger oscillation in system output compared to previous case. This also can be seen in its tracking error in the bottom graphs since bigger distortion turns out in some parts of the error signal yielded by backstepping-GSA. Integration of backstepping with PSO creates smooth output with zero tracking error.

Case 3. In this case, time-varying signal replaced signal disturbance to the system output. Figure 4 shows system output with respect to reference input given and tracking error between reference input and system output for both incorporation of backstepping with GSA and PSO, respectively.

Similar as previous case, when time-varying signal perturbed the system's actuator, although the system output with backstepping-GSA follows reference input given, there is oscillation when the reference input changes its form. This situation is also can be seen in its tracking error which produces bigger spike and oscillation compared to the output in Case 2. Combination of backstepping with PSO still produces smooth response with zero-tracking error in this case.

Case 4. System without disturbance in this case plays as benchmark for the three other cases. System output with respect to reference input given and its tracking error for each integration of backstepping with GSA and PSO can be seen in Figure 5.

Without any disturbance given to system's actuator, both assimilation of backstepping with GSA and PSO generate 
Position output, $x_{1}$ with respect to

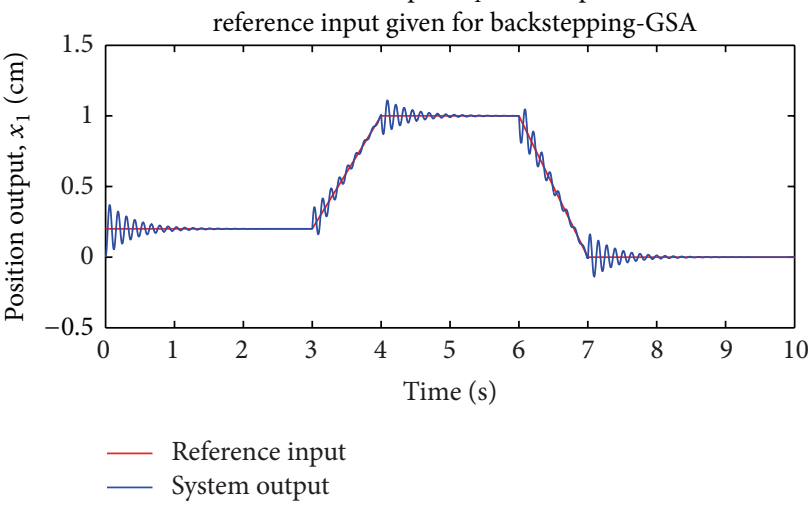

(a)

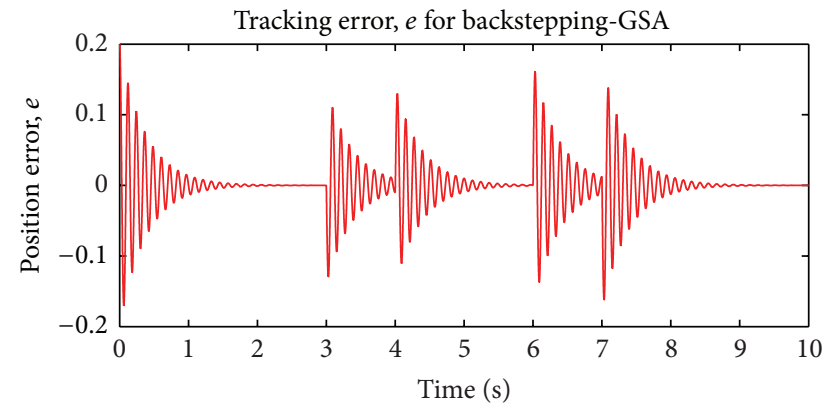

(c)

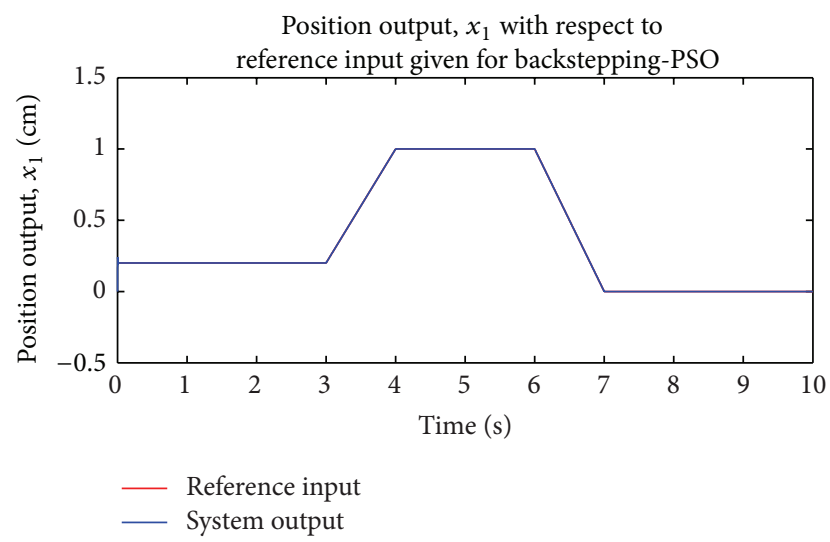

(b)

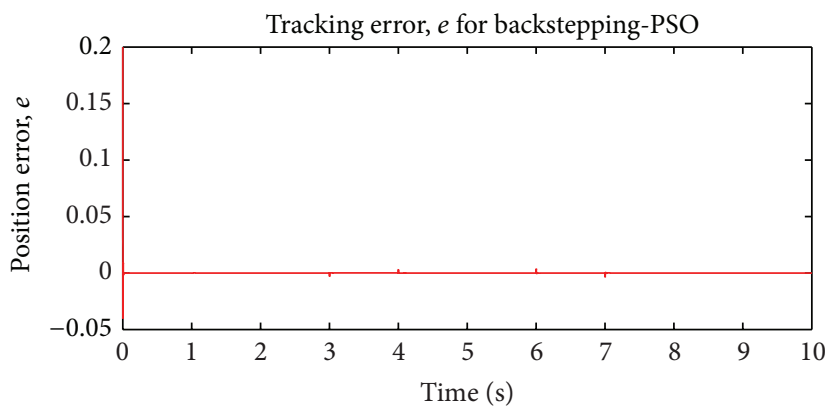

(d)

FIGURE 3: Position output with respect to reference input given and tracking error for backstepping-PSO and backstepping-GSA with step disturbance.

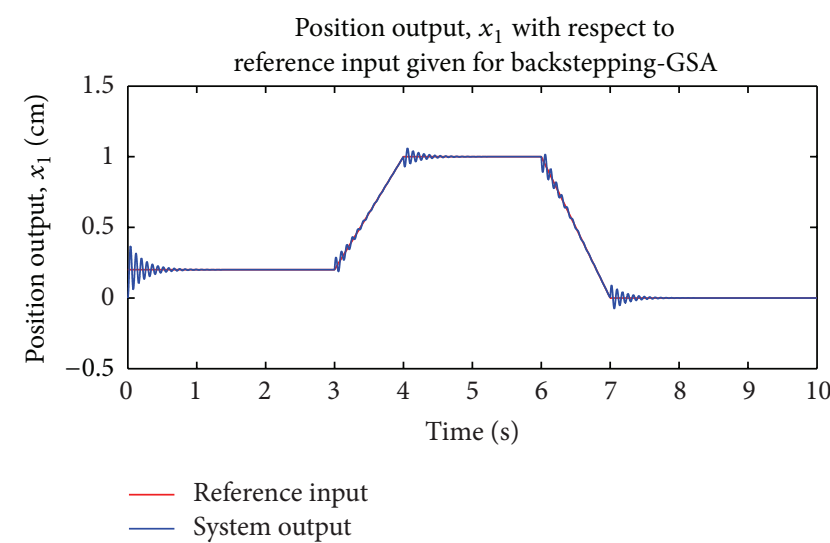

(a)

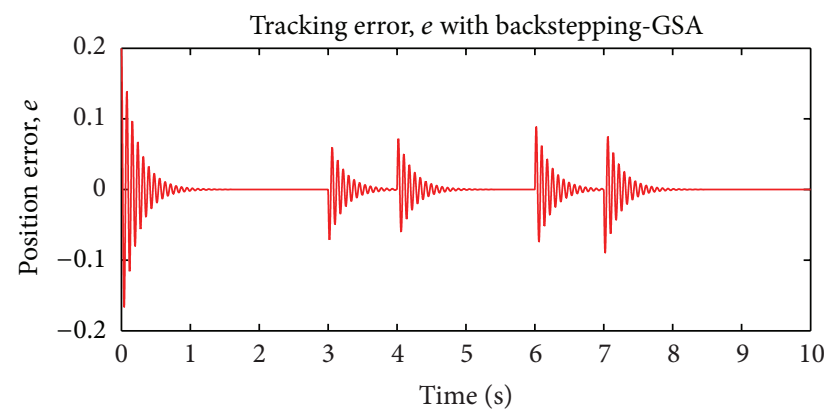

(c)

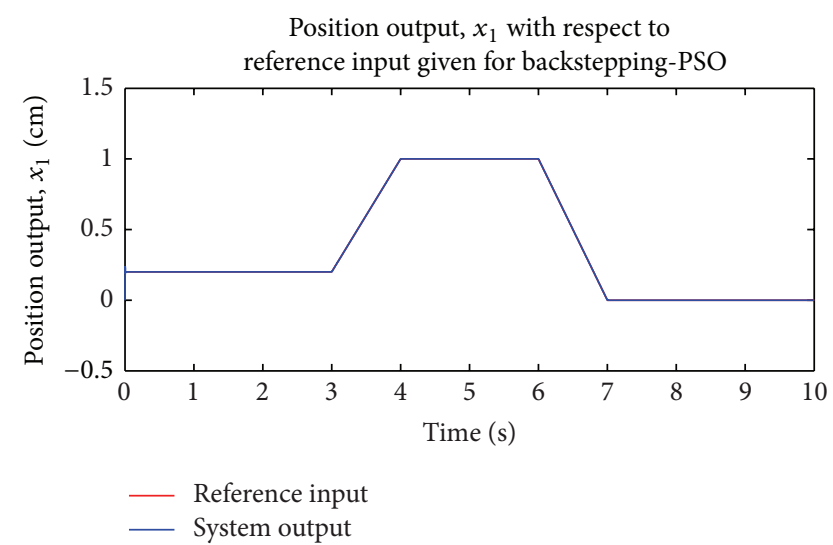

(b)

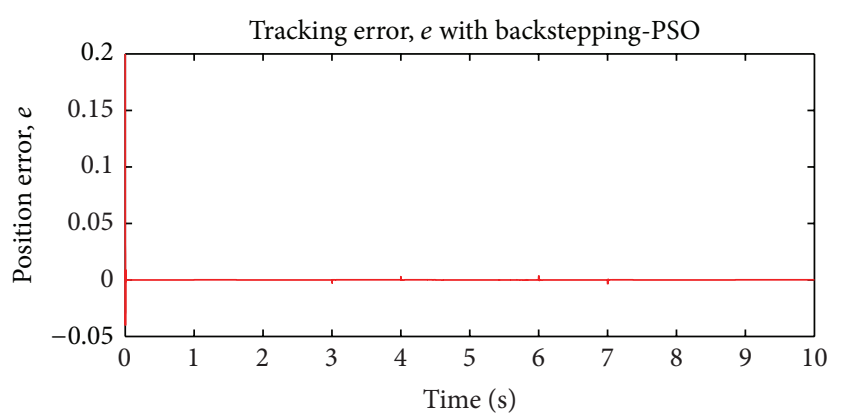

(d)

FIGURE 4: Position output with respect to reference input given and tracking error for backstepping-PSO and backstepping-GSA with timevarying disturbance. 


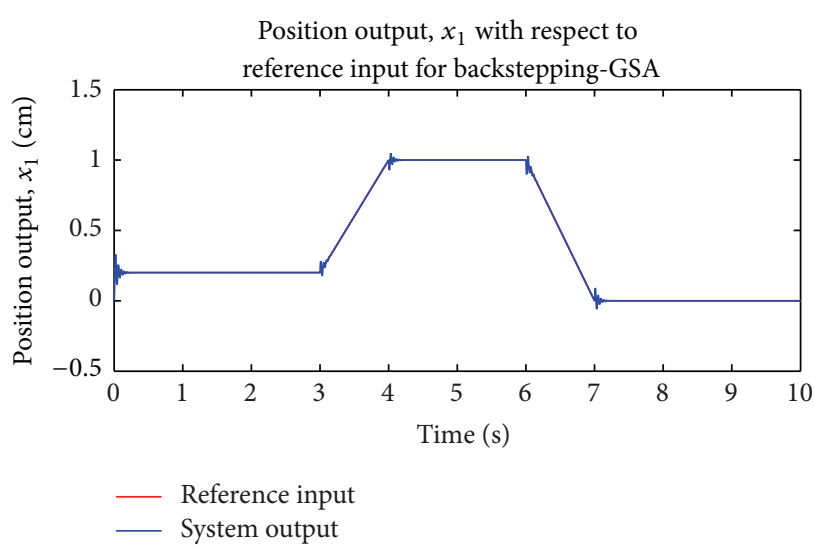

(a)

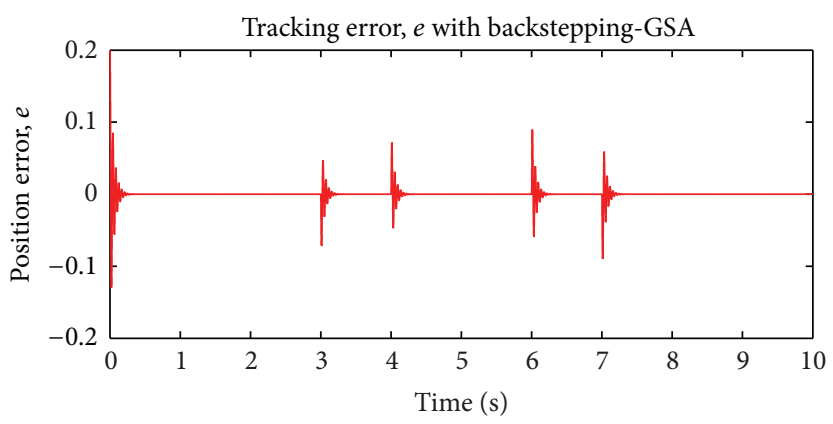

(c)

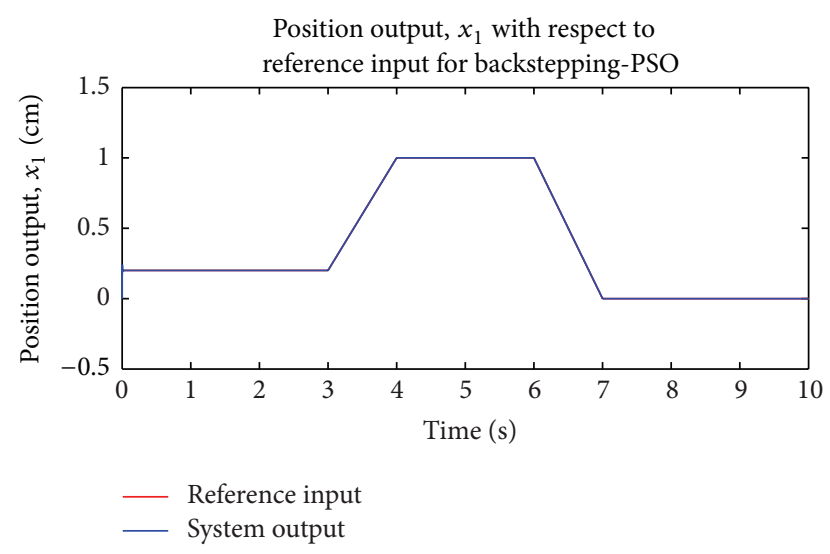

(b)

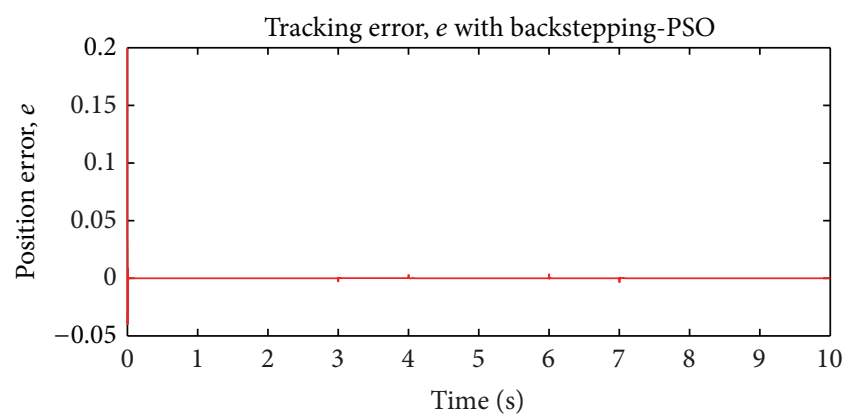

(d)

FIGURE 5: Position output with respect to reference input given and tracking error for backstepping-PSO and backstepping-GSA without disturbance.

TABLE 2: Parameters of backstepping controller obtained from PSO and GSA techniques for each case.

\begin{tabular}{|c|c|c|c|c|c|c|c|c|c|c|c|c|}
\hline \multirow{2}{*}{ Case } & \multicolumn{6}{|c|}{ PSO } & \multicolumn{6}{|c|}{ GSA } \\
\hline & $k_{1}$ & $k_{2}$ & $k_{3}$ & $\rho_{1}$ & $\rho_{2}$ & $\rho_{3}$ & $k_{1}$ & $k_{2}$ & $k_{3}$ & $\rho_{1}$ & $\rho_{2}$ & $\rho_{3}$ \\
\hline Case 1 & 698.86 & 1 & 1 & 1 & 1500 & 1 & 1178.9 & 177.39 & 37.2 & 1488.6 & 1006.9 & 23.69 \\
\hline Case 2 & 1500 & 1500 & 1500 & 1500 & 1 & 1 & 315.36 & 979.28 & 1031.7 & 728.82 & 1135.5 & 56.5 \\
\hline Case 3 & 1500 & 1 & 832.60 & 1 & 1500 & 1 & 132 & 1405.9 & 717.29 & 328.17 & 993.25 & 15.26 \\
\hline Case 4 & 1500 & 30.87 & 830.98 & 1500 & 1500 & 1 & 1397.3 & 174.53 & 838.82 & 1191.4 & 1244.7 & 15.56 \\
\hline
\end{tabular}

smooth and steady output response with respect to reference input given. The tracking error with these two controllers also zeros. However, based on Figure 5, there is small distortion in the tracking error of backstepping-GSA caused by changing of shape of the reference input.

Performance of chosen system with the designed backstepping controller depends on the control parameters, $k_{1}, k_{2}$, $k_{3}, \rho_{1}, \rho_{2}$, and $\rho_{3}$ which are obtained through PSO and GSA techniques. Table 2 revealed the parameters of the designed controller yielded from these two different optimization techniques for each case.

All these control parameters are obtained through PSO and GSA techniques by giving the system tracking error to both algorithms. These algorithms will operate in order to minimize its objective function, SSE. Table 3 explained SSE obtained from each combination of backstepping with PSO and GSA.
TABLE 3: SSE obtained from combination of backstepping with PSO and GSA.

\begin{tabular}{lcc}
\hline & \multicolumn{2}{c}{ Sum of squared error, SSE } \\
& PSO & GSA \\
\hline Case 1 & 0.8407 & 33.1103 \\
Case 2 & 15.0156 \\
Case 3 & 0.5533 & 52.1216 \\
Case 4 & 0.5765 & 4.3118 \\
\hline
\end{tabular}

Based on Table 3, integration of backstepping with PSO yields smaller SSE as compared to its combination with GSA, although different type of disturbance signal is given to the system's actuator. 


\section{Conclusion}

In this research work, electrohydraulic actuator system is chosen as an example since its position tracking is highly nonlinear. External force is given as perturbation to the actuator of the system. Different type of signal is given as disturbance to the system. Backstepping controller is designed for the system in simulation environment by considering the system set up with and without disturbance. In the process of designing this controller, several parameters are produced. The determination of these parameters is important in order to obtain good performance of the controller for the system. Particle swarm optimization (PSO) and gravitational search algorithm (GSA) techniques are chosen as a tool to optimise controller parameters. Simulation results show that combination of PSO with backstepping is better than integration of backstepping with GSA. Performance results show that backstepping-PSO produces smooth output response and smaller tracking error compared to backstepping-GSA. Robustness of the designed controller is also tested by giving different type of disturbance signal.

\section{Conflict of Interests}

The authors declare that there is no conflict of interests regarding the publication of this paper.

\section{References}

[1] A. Witkowska, M. Tomera, and R. Smierzchalski, "A backstepping approach to ship course control," International Journal of Applied Mathematics and Computer Science, vol. 17, no. 1, pp. 7385, 2007.

[2] K. Ioannis Kristic, M. I. Kanellakoupulos, and P. V. Kokotovic, Nonlinear and Adaptive Control Design, John Wiley \& Sons, New York, NY, USA, 1995.

[3] H. K. Khalil, Nonlinear Systems, Prentice Hall, Upper Saddle River, NJ, USA, 3rd edition, 2002.

[4] E. Zergeroglu, Y. Fang, M. S. Queiroz, and D. M. Dawson, "Global output feedback control of dynamically positioned surface vessels: an adaptive control approach," Mechatronics, vol. 14, no. 4, pp. 341-356, 2004.

[5] P. Nakkarat and S. Kuntanapreeda, "Observer-based backstepping force control of an electrohydraulic actuator," Control Engineering Practice, vol. 17, no. 8, pp. 895-902, 2009.

[6] B. M. Dehkordi and A. Farrokh Payam, "Nonlinear slidingmode controller for sensorless speed control of DC servo motor using adaptive backstepping observer," in Proceedings of the International Conference on Power Electronics, Drives and Energy Systems (PEDES '06), pp. 1-5, New Delhi, India, December 2006.

[7] C. Kaddissi, J.-P. Kenne, and M. Saad, "Drive by wire control of an electro-hydraulic active suspension a backstepping approach," in Proceedings of the IEEE Conference On Control Applications (CCA '05), pp. 1581-1587, IEEE, Toronto, Canada, August 2005.

[8] A. Karimi, A. Al-Hinai, K. Schoder, and A. Feliachi, "Power system stability enhancement using backstepping controller tuned by particle swarm optimization technique," in Proceedings of the IEEE Power Engineering Society General Meeting, vol. 2, pp. 1388-1395, San Francisco, Calif, USA, June 2005.
[9] H. Yu, Z.-J. Feng, and X.-Y. Wang, "Nonlinear control for a class of hydraulic servo system," Journal of Zhejiang University: Science A, vol. 5, no. 11, pp. 1413-1417, 2004.

[10] M. R. Sirouspour and S. E. Salcudean, "On the nonlinear control of hydraulic servo-systems," in Proceedings of the IEEE International Conference on Robotics and Automation (ICRA '00), vol. 2, pp. 1276-1282, San Francisco, Calif, USA, April 2000.

[11] K. Queiroz, A. D. Araujo, M. V. A. Fernandes, S. M. Dias, and J. B. Oliveira, "Variable structure adaptive backstepping controller for plants with arbitrary relative degree based on modular design," in Proceedings of the American Control Conference (ACC '10), pp. 3241-3246, IEEE, Baltimore, Md, USA, July 2010.

[12] Q. Wang, Z. Liu, and L. Er, "Disturbance observer based robust backstepping control for flight simulator," in Proceedings of the Multiconference on Computational Engineering in Systems Applications (CESA '06), pp. 1366-1370, IEEE, Beijing, China, October 2006.

[13] J. M. Lee, H. M. Kim, S. H. Park, and J. S. Kim, "A position control of electro-hydraulic actuator systems using the adaptive control scheme," in Proceedings of the 7th Asian Control Conference (ASCC '09), pp. 21-26, IEEE, Hong Kong, China, August 2009.

[14] T. Zhang, S. S. Ge, and C. C. Hang, "Adaptive neural network control for strict-feedback nonlinear systems using backstepping design," Automatica, vol. 36, no. 12, pp. 1835-1846, 2000.

[15] I. Ursu, F. Ursu, and F. Popescu, "Backstepping design for controlling electrohydraulic servos," Journal of the Franklin Institute, vol. 343, no. 1, pp. 94-110, 2006.

[16] M. Smaoui, X. Brun, and D. Thomasset, "A study on tracking position control of an electropneumatic system using backstepping design," Control Engineering Practice, vol. 14, no. 8, pp. 923933, 2006.

[17] T. K. Roy, "Adaptive backstepping controller for altitude control of a small scale helicopter by considering the ground effect compensation," in Proceedings of the International Conference on Informatics, Electronics \& Vision (ICIEV '13), pp. 1-5, Dhaka, India, May 2013.

[18] N. Adhikary and C. Mahanta, "Integral backstepping sliding mode control for underactuated systems: swing-up and stabilization of the Cart-Pendulum System," ISA Transactions, vol. 52, no. 6, pp. 870-880, 2013.

[19] J. Ye, "Tracking control for nonholonomic mobile robots: integrating the analog neural network into the backstepping technique," Neurocomputing, vol. 71, no. 16-18, pp. 3373-3378, 2008.

[20] Y. Al-Younes and M. A. Jarrah, "Attitude stabilization of quadrotor UAV using backstepping fuzzy logic \& backstepping leastmean-square controllers," in Proceedings of the 5th International Symposium on Mechatronics and Its Applications (ISMA '08), pp. 1-11, IEEE, Amman, Jordan, May 2008.

[21] C.-K. Chen, W.-Y. Wang, Y.-G. Leu, and C.-Y. Chen, "Compact ant colony optimization algorithm based fuzzy neural network backstepping controller for MIMO nonlinear systems," in Proceedings of the International Conference on System Science and Engineering (ICSSE '10), pp. 146-149, IEEE, Taipei, Taiwan, July 2010.

[22] C.-Y. Li, W.-X. Jing, and C.-S. Gao, "Adaptive backsteppingbased flight control system using integral filters," Aerospace Science and Technology, vol. 13, no. 2-3, pp. 105-113, 2009.

[23] A. Karimi and A. Feliachi, "PSO-tuned adaptive backstepping control of power systems," in Proceedings of the IEEE PES Power 
Systems Conference and Exposition (PSCE '06), pp. 1315-1320, IEEE, Atlanta, Ga, USA, November 2006.

[24] R. J. Wai and K. L. Chuang, "Design of backstepping particleswarm-optimisation control for maglev transportation system," IET Control Theory \& Applications, vol. 4, no. 4, pp. 625-645, 2010.

[25] F.-J. Lin and S.-Y. Y. Chen, "Intelligent integral backstepping sliding mode control using recurrent neural network for magnetic levitation system," in Proceedings of the International Joint Conference on Neural Networks (IJCNN '10), pp. 1-7, IEEE, Barcelona, Spain, July 2010.

[26] M. Yang, X. Wang, and K. Zheng, "Nonlinear controller design for permanent magnet synchronous motor using adaptive weighted PSO," in Proceedings of the American Control Conference (ACC '10), pp. 1962-1966, IEEE, Baltimore, Md, USA, July 2010.

[27] X. Lin, Y. Xie, D. Zhao, and S. Xu, "Acceleration feedback adaptive backstepping controller for DP using PSO," in Proceedings of the 9th International Conference on Fuzzy Systems and Knowledge Discovery (FSKD '12), pp. 2334-2338, IEEE, Sichuan, China, May 2012.

[28] R.-C. David, R.-E. Precup, S. Preitl, J. K. Tar, and J. Fodor, "Parametric sensitivity reduction of PI-based control systems by means of evolutionary optimization algorithms," in Proceedings of the 6th IEEE International Symposium on Applied Computational Intelligence and Informatics (SACI '11), pp. 241-246, IEEE, Timisoara, Romania, May 2011.

[29] S. Duman, M. Dincer, and G. Ugur, "Determination of the PID controller parameters for speed and position control of DC motor using gravitational search algorithm," in Proceedings of the 7th International Conference on Electrical and Electronics Engineering (ELECO '11), pp. I-225-I-229, IEEE, Bursa, Turkey, December 2011.

[30] R.-E. Precup, "Novel adaptive gravitational search algorithm for fuzzy controlled servo systems," IEEE Transactions on Industrial Informatics, vol. 8, no. 4, pp. 791-800, 2011.

[31] A. S. Rostami, B. H. Mehrpour, and A. R. Hosseinabadi, "A novel and optimized Algorithm to select monitoring sensors by GSA," in Proceedings of the International Conference on Control, Instrumentation and Automation (ICCIA '11), pp. 829834, IEEE, Shiraz, Iran, December 2011.

[32] S. H. Zahiri, "Fuzzy gravitational search algorithm an approach for data mining," Iranian Journal of Fuzzy Systems, vol. 9, no. 1, pp. 21-37, 2012.

[33] M. Khajehzadeh, M. R. Taha, A. El-Shafie, and M. Eslami, "A modified gravitational search algorithm for slope stability analysis," Engineering Applications of Artificial Intelligence, vol. 25, no. 8, pp. 1589-1597, 2012.

[34] A. P. Engelbrecht, Computational Swarm Intelligence: An Introduction, John Wiley \& Sons, Chichester, UK, 2nd edition, 2007.

[35] E. Rashedi, H. Nezamabadi-pour, and S. Saryazdi, "GSA: a gravitational search algorithm," Information Sciences, vol. 179, no. 13, pp. 2232-2248, 2009. 


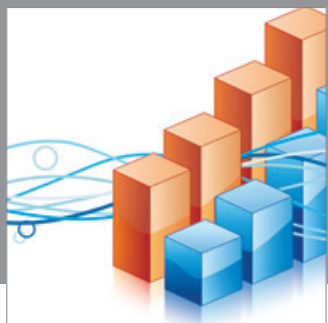

Advances in

Operations Research

mansans

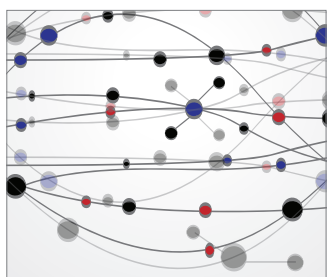

The Scientific World Journal
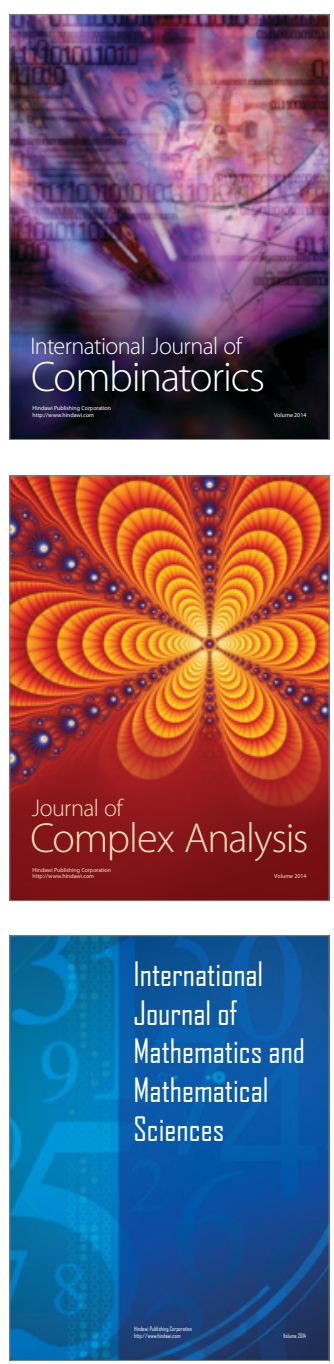
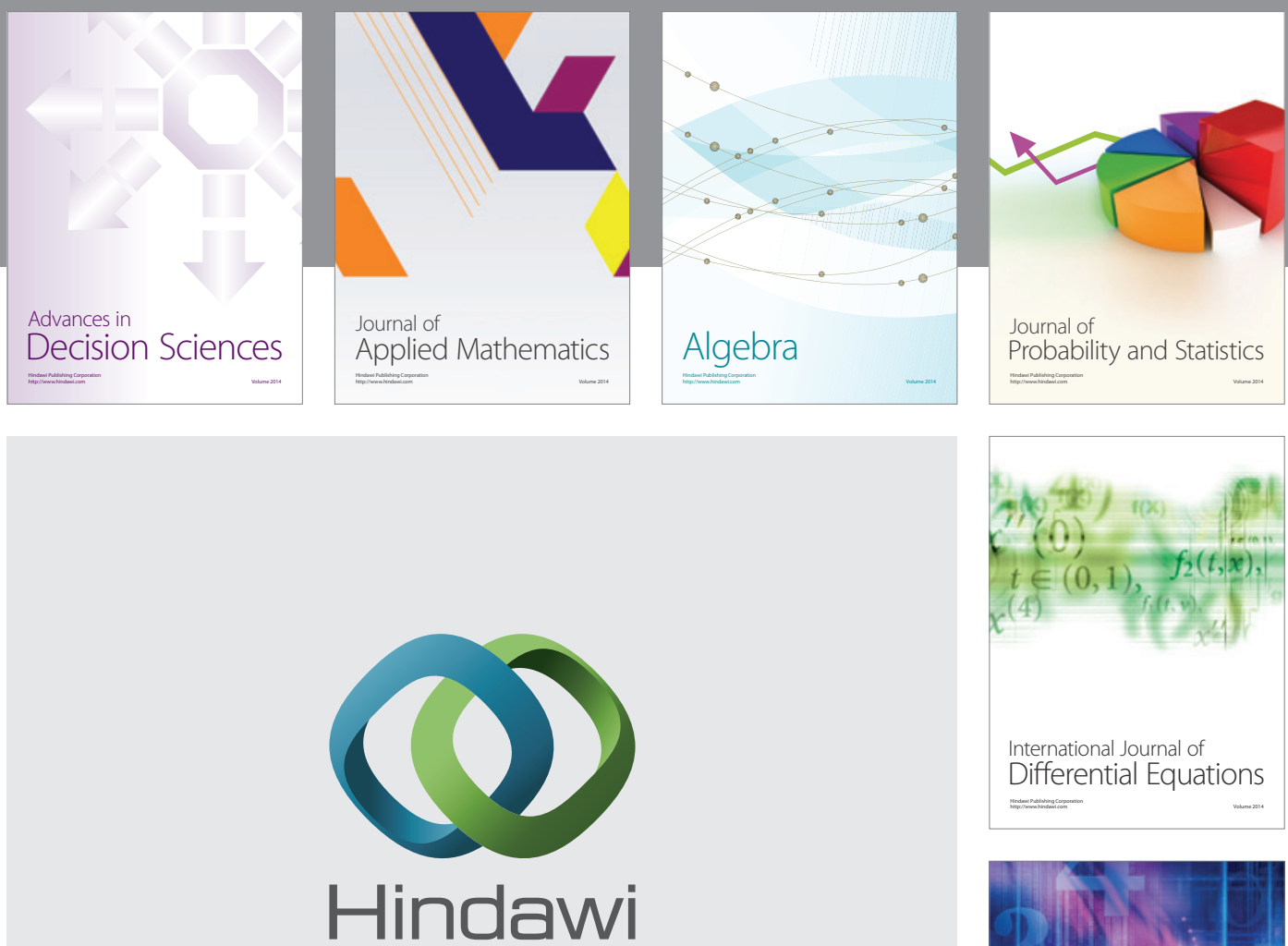

Submit your manuscripts at http://www.hindawi.com
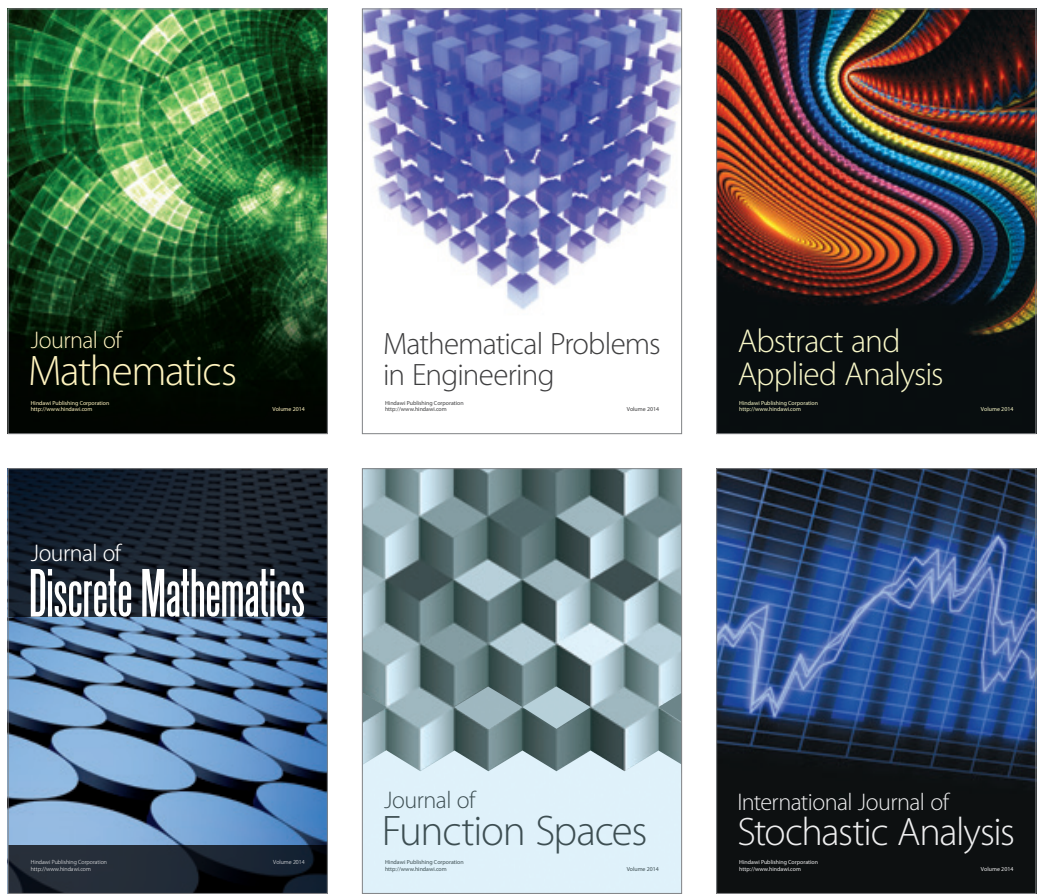

Journal of

Function Spaces

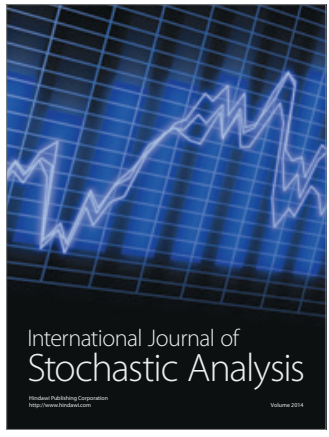

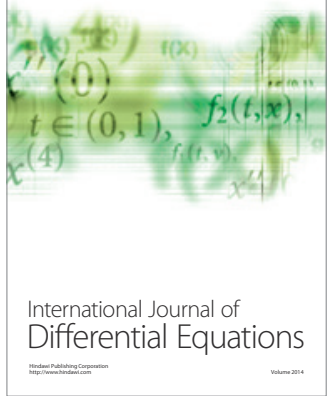
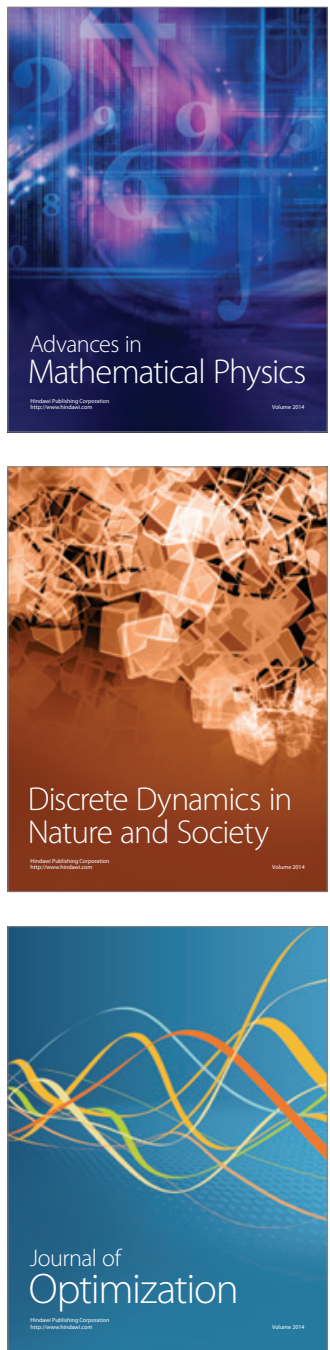